\title{
Crescimento e sobrevivência de larvas do jundiá, Rhamdia quelen, alimentadas com alimento vivo enriquecido e dieta artificial*
}

\section{Growth and survival of silver catfish, $R$. quelen, larvae fed enriched live food and artificial diet}

\author{
Pedro Gusmão Borges Neto, ${ }^{* *}$ Fabricio Martins Dutra, ${ }^{* * *}$ Eduardo Luis Cupertino Ballester, ${ }^{* * *}{ }^{2}$ Leandro Portz ${ }^{* * *}$
}

\begin{abstract}
Resumo
O objetivo do presente estudo foi avaliar a utilização de dieta inerte e do alimento vivo Artemia salina durante a larvicultura do jundiá Rhamdia quelen. As larvas foram alimentadas de acordo com os seguintes tratamentos: náuplios de $A$. salina (T1), metanáuplios (T2), metanáuplios enriquecidos com ácidos graxos (T3), metanáuplios enriquecidos com ácidos graxos e vitamina C (T4) e dieta artificial (T5). O delineamento foi inteiramente casualizado com cinco tratamentos e quatro repetições $(5 \times 4)$. As larvas foram alimentadas quatro vezes ao dia, em densidade de estocagem de 10 larvas por litro. Os parâmetros de desempenho utilizados foram sobrevivência, ganho de peso, crescimento final e desenvolvimento morfológico. Ao fim do experimento, considerando os resultados obtidos, pôde-se concluir que os tratamentos com o uso de $A$. salina foram satisfatórios, apresentando sobrevivência acima de $80 \%$ e bons índices de desempenho, atingindo peso médio final de $0.011 \pm 0.004 \mathrm{~g}$ e comprimento médio final de 7.150 $\pm 1.02 \mathrm{~mm}(\mathrm{P}<0,05)$ para o tratamento com náuplios de Artemia. A dieta inerte utilizada não é recomendada para a larvicultura do jundiá, favorecendo o canibalismo e reduzindo significativamente a sobrevivência e crescimento.
\end{abstract}

Palavras-chave: aquicultura, larvicultura, jundiá, Artemia, desempenho produtivo.

\begin{abstract}
The aim of this study was to evaluate the use of inert and live food diet Artemia salina during larval rearing of catfish $R$. quelen. The larvae were fed according to the following treatments: Artemia saline nauplii (T1), metanauplii (T2), metanauplii enriched with fatty acids (T3), metanauplii enriched with fatty acids and vitamin C (T4) and artificial diet (T5). The design was completely randomized with five treatments and four replications $(5 \times 4)$. The larvae were fed four times daily at a stocking density of 10 larvae per liter. Performance parameters used were survival, weight gain, the final growth and morphological development. At the end of the experiment, considering the results obtained, it can be concluded that the treatment with the use of $A$. saline were satisfactory, with over $80 \%$ survival and good performance indexes reaching final average weight of $0.011 \pm 0.004 \mathrm{~g}$ and length $7.150 \pm 1.02$ $\mathrm{mm}(\mathrm{P}<0.05)$ for treatment with Artemia. The inert diet used is not recommended for larval rearing of catfish, favoring cannibalism and significantly reduced survival and growth.
\end{abstract}

Keywords: aquaculture, hatchery, catfish, brine shrimp, productive performance.

\section{Introdução}

O jundiá, Rhamdia quelen, é uma espécie nativa da família Heptapteridae, com distribuição neotropical e ocorrência registrada desde o México até o sul da Argentina (Silfvergrip, 1996).

No Brasil, R. quelen é uma das mais produzidas na região Sul, sendo caracterizado pelo hábito alimentar onívoro, facilidade de reprodução e aceitabilidade pelo consumidor (Radünz-Neto, 1981; Gomes et al., 2000).

Embora sua produção encontre-se em franco desenvolvimento, a larvicultura do jundiá ainda apresenta alguns entraves, a exemplo do canibalismo e consequente crescimento desuniforme. Uma alternativa para contornar tal dificuldade é o fornecimento de alimento de qualidade durante os primeiros dias de vida em laboratório.
Em linhas gerais, o regime alimentar na larvicultura de peixes conduzida em laboratório consiste no fornecimento de organismos alimento, a exemplo de cladóceros, copepódos e rotíferos, dietas artificiais ou a combinação destas (Luz e Portella, 2005; Luz e Zaniboni Filho, 2008 e Wachter et al., 2009). Neste sistema, vantagens e desvantagens no uso de dietas artificiais ou alimento vivo são observadas durante a larvicultura, normalmente associadas à capacidade de cada espécie em digerir e assimilar os nutrientes.

Durante esta fase inicial de desenvolvimento, diversos fatores influenciam no crescimento e sobrevivência de larvas, a exemplo do fotoperíodo (Feiden et al., 2006), cor dos tanques de larvicultura (Pedreira et al., 2008) e tamanho do alimento fornecido (Côrtes e Tsuzuki, 2010).

\footnotetext{
*Recebido em 2 de outubro de 2013 e aceito em 20 de dezembro de 2013.

${ }^{* *}$ Professor Substituto da Universidade Federal do Paraná - UFPR. Setor Palotina, Rua Pioneiro, 2153 - Jardim Dallas - CEP 85950-000 - Palotina Paraná - Brasil. E-mail: pgusmao@ufpr.br.

***Doutorando do Programa de Pós-Graduação em Zoologia - Departamento de Ciências Biológicas - Universidade Federal do Paraná.

****Professor Doutor - Setor Palotina - Universidade Federal do Paraná.
} 
Apesar dos avanços recentes na nutrição de larvas, dificuldades em determinar exigências nutricionais e identificar a influência de possíveis mudanças durante o processo ontogênico ainda persistem para jundiá e para a maioria das espécies cultivadas e de potencial zootécnico (Aragão et al., 2004).

Embora dietas artificiais apresentem benefícios relacionados com a estabilidade e balanço de nutrientes (Dias, 1989), a incapacidade morfofisiológica de larvas de peixes em digerir moléculas complexas durante o processo de formação do trato digestório, logo após início da alimentação exógena, pode ser um entrave na utilização de dietas artificiais (Holt, 2011).

O elevado padrão nutritivo do alimento vivo utilizado na aquicultura, a exemplo de náuplios e metanáuplios de Artemia salina, torna-o indispensável para sobrevivência de pós-larvas e juvenis de peixes cultivados (Ronnestad et al., 1999).

Ademais, a possibilidade de enriquecimento deste microcrustáceo com vitaminas, ácidos graxos, proteína e outros nutrientes (Agh e Sorgeloos, 2005), através do processo de bioencapsulação, aperfeiçoa o aproveitamento de insumos e reduz impactos de processos extensivos de larvicultura.

Diante do exposto, o presente estudo objetivou avaliar a utilização de alimento vivo $A$. salina, sob a forma de náuplios recém-eclodidos e metanáuplios de Artemia não enriquecidos e enriquecidos com ácidos graxos e vitamina $\mathrm{C}$, em relação à dieta artificial durante a fase inicial da larvicultura do jundiá, $R$. quelen.

\section{Materiais e métodos}

O presente estudo foi realizado no Laboratório de Aquariologia e Produção de Organismos Alimento da Universidade Federal do Paraná - Setor Palotina. Para tanto, cerca de 5000 ovos de jundiá, $R$. quellen, foram obtidos a partir de reprodutores da espécie seguindo o protocolo de reprodução e indução hormonal (Ihering, 1937; Zaniboni Filho e Barbosa 1996), e posteriormente acondicionados em incubadora tipo funil $(60 \mathrm{~L})$, até o momento da eclosão.

As larvas do jundiá permaneceram na incubadora por um período pre-determinado de 50 horas, suficiente para absorção parcial do saco vitelínico e abertura da boca (Pereira et al., 2006). Após verificação em estereomicroscópio da abertura de boca e capacidade de ingerir alimento exógeno, as larvas foram contadas individualmente e estocadas na densidade de 10 larvas por litro em aquários, com 20 litros de volume utilizável, totalizando 200 larvas por unidade experimental.

Após iniciado o experimento, as larvas foram alimentadas de acordo com os seguintes tratamentos: náuplios de Artemia salina recém-eclodidos (T1), metanáuplios de $A$. salina (T2), metanáuplios de $A$. salina enriquecidos com ácidos graxos (T3), metanáuplios de $A$. salina enriquecidos com ácidos graxos e vitamina C (T4) e dieta artificial (T5), em delineamento inteiramente casualizado (DIC), com cinco tratamentos e quatro repetições $(5 \times 4)$.

Os náuplios e metanáuplios de Artemia utilizados neste estudo foram obtidos através da eclosão de cistos oriundos do Great Salt Lake - INVE ${ }^{\circledR}$, seguindo o protocolo descrito por Lavens e Sorgeloos (1996). Após realizado o processo de eclosão, os náuplios recém-eclodidos foram coletados e ofertados às larvas de jundiá juntamente com os metanáuplios enriquecidos.

Para o enriquecimento dos metanáuplios de $A$. salina com ácidos graxos e vitamina $C$, seguiu-se o conjunto de técnicas descritas por Agh e Sorgeloos (2005). O processo de enriquecimento consistiu em manter os metanáuplios em recipientes contendo uma solução de enriquecimento, para assimilação de nutrientes.

A solução de enriquecimento utilizada, composta por ácidos graxos poli-insaturados, foi a Super Selco - INVE ${ }^{\circledR}$ na proporção de um grama para cada litro de água com densidade máxima de 300 metanáuplios por mililitro. Para o tratamento com adição de vitamina C (Stay C - Roche ${ }^{\circledR}$ ), foi realizada a substituição de $15 \%$ de cada grama do enriquecedor de ácidos graxos por esta vitamina.

O manejo alimentar em todos os tratamentos com inclusão de $A$. salina na forma de náuplios e metanáuplios foi baseado no fornecimento de 400 organismos por larva de jundiá nos primeiros cinco dias de experimento e 600 organismos por larva no período posterior. A frequência de alimentação adotada foi de cinco vezes ao longo do dia, sendo duas pela manhã, às 8 horas e 11 horas, duas à tarde, às 13 horas e 17 horas, e uma à noite, às 19 horas.

A dieta artificial comercial Anhambi $^{\circledR}$ (Tabela 1) utilizada no tratamento cinco continha cerca de $40 \%$ de proteína bruta e 4000 kcal de energia bruta.

Tabela 1: Composição da ração artificial utilizada na alimentação de larvas do jundiá, $R$. quelen

\begin{tabular}{lc}
\hline Componente & Proporção \\
\hline Proteína bruta & $400 \mathrm{~g} / \mathrm{kg}$ \\
Extrato etéreo & $80 \mathrm{~g} / \mathrm{kg}$ \\
Matéria fibrosa & $40 \mathrm{~g} / \mathrm{kg}$ \\
Matéria mineral & $130 \mathrm{~g} / \mathrm{kg}$ \\
Cálcio & $15 / 38 \mathrm{~g} / \mathrm{kg}$ \\
Fósforo & $12 \mathrm{~g} / \mathrm{kg}$ \\
Umidade & $120 \mathrm{~g} / \mathrm{kg}$ \\
\hline
\end{tabular}

O processo de alimentação das larvas com dieta artificial consistiu em umedecer a ração comercial em pó para que esta adquirisse textura pastosa. A taxa de arraçoamento foi equivalente à proporção de náuplios e metanáuplios de $A$. salina utilizados, definida por relação de equivalência entre peso da biomassa de Artêmia utilizada e dieta inerte. A frequência alimentar adotada foi equivalente à definida para o alimento vivo utilizado.

A higienização do sistema experimental foi realizada diariamente através da remoção de sólidos por sifonamento do fundo do aquário, realizado antes do último período de alimentação, e troca parcial de água, realizada durante toda a larvicultura, representando cerca de $50 \%$ do volume total de cada unidade experimental.

No controle do fotoperíodo, a intensidade luminosa foi reduzida para inibir o canibalismo entre larvas do jundiá (Behr et al., 1999), sendo adotado o regime de penumbra com níveis de intensidade luminosa abaixo de 10 lux durante o dia, monitorado por Luximetro (Instrutherm - LD 209), sendo a luz ausente durante a noite.

Para coleta de dados biométricos, uma amostra inicial de 200 larvas foi coletada do grupo de larvas da incubadora, sendo aferidos e registrados valores de peso (gramas) e comprimento total (milímetros). Da mesma forma, ao fim do experimento, após 
15 dias do início, houve coleta de dados biométricos, peso e comprimento, de todos os indivíduos utilizados no experimento. Os animais foram sacrificados por termonarcose.

As imagens capturadas de larvas, oriundas da biometria inicial e de cada tratamento ao fim do experimento, foram obtidas por microscopia óptica através de câmera Leica DFC 500 e do software Auto - Montage Pro (Syncropy ${ }^{\circledR}$ ).

O monitoramento da qualidade de água foi realizado diariamente para o $\mathrm{pH}$, oxigênio dissolvido e temperatura. A cada dois dias foram aferidos os valores de amônia, alcalinidade e dureza.

As análises estatísticas dos dados foram realizadas no software computacional Statistica $7.0^{\circledR}$, sendo aplicada ANOVA para análise de variância e teste de Tukey para comparação de médias entre tratamentos.

\section{Resultados}

As análises da qualidade de água (Tabela 2) revelaram uma variação normal entre todos os tratamentos para os parâmetros de alcalinidade, dureza, oxigênio dissolvido, temperatura e pH. No entanto, a análise de produtos nitrogenados revelou um aumento na concentração de amônia total para o tratamento cinco (T5) com uso de dieta artificial.

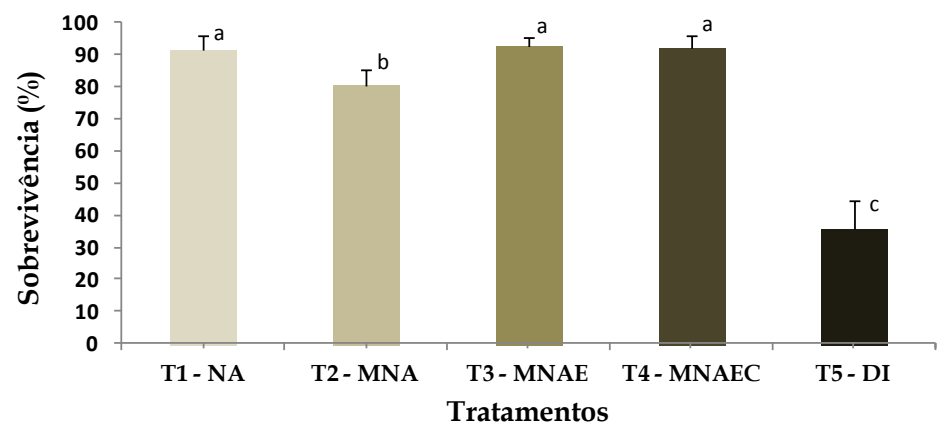

Figura 1: Sobrevivência de pós-larvas do Rhamdia quelen alimentadas com Artemia salina e dieta inerte. Legenda: (T1 - náuplios de Artemia, T2 Metanáuplios Artemia, T3 - Metanáuplios de Artemia enriquecidos com ácidos graxos, T4 - Metanáuplios de Artemia enriquecidos com ácidos graxos e Vitamina C, T5 - Dieta inerte ou artificial).

o período experimental comparativamente ao tratamento com metanáuplios não enriquecidos. Por sua vez, o uso de Artemia na forma de náuplios recém eclodidos promoveu sobrevivência superior a $90 \%$.

Em relação ao desenvolvimento das larvas, o ganho de peso variou significativamente de acordo com o tipo de alimento utilizado nos diferentes tratamentos, como pode ser observado na Figura 2.

Tabela 2: Variáveis de qualidade de água e variação de acordo com os tratamentos adotados na larvicultura do jundiá, R. quelen

\begin{tabular}{|c|c|c|c|c|c|c|}
\hline Tratamento & $\begin{array}{c}\text { Alcalinidade } \\
\mathrm{mg} \cdot \mathrm{L}^{-1} \mathrm{de} \mathrm{CaCo}^{3 * *}\end{array}$ & $\begin{array}{c}\text { Dureza } \\
\mathrm{mg} \cdot \mathrm{L}^{-1} \mathrm{de} \mathrm{CaCo}^{3 *}\end{array}$ & $\begin{array}{c}\text { Oxigênio } \\
\text { mg. } \mathrm{L}^{-1 *}\end{array}$ & $\begin{array}{l}\text { Amônia } \\
\mathrm{mg} \cdot \mathrm{L}^{-1 * *}\end{array}$ & $\begin{array}{c}\text { Temperatura } \\
{ }^{\circ} \mathrm{C}^{*} \\
\end{array}$ & $\mathrm{pH}^{*}$ \\
\hline 1- NA & $52.0 \pm 7.8^{b}$ & $15.5 \pm 3.0$ & $6.6 \pm 0.3$ & $0.1 \pm 0.1^{\mathrm{b}}$ & $29.3 \pm 0.3$ & $7.9 \pm 0.1$ \\
\hline T2-MNA & $54.0 \pm 7.1^{b}$ & $18.0 \pm 1.6$ & $6.7 \pm 0.1$ & $0.1 \pm 0.1^{b}$ & $28.4 \pm 0.3$ & $8.2 \pm 0.5$ \\
\hline T3-MNAE & $52.5 \pm 5.9^{b}$ & $14.0 \pm 2.8$ & $6.3 \pm 0.2$ & $0.1 \pm 0.2^{\mathrm{b}}$ & $29.3 \pm 0.3$ & $7.9 \pm 0.1$ \\
\hline T4-MNAEC & $55.5 \pm 3.7^{b}$ & $14.0 \pm 1.6$ & $6.5 \pm 0.1$ & $0.1 \pm 0.1^{b}$ & $29.7 \pm 0.2$ & $8.1 \pm 0.1$ \\
\hline T5-DI & $58.0 \pm 5.4^{\mathrm{a}}$ & $16.5 \pm 1.9$ & $5.7 \pm 0.2$ & $0.7 \pm 0.2^{\mathrm{a}}$ & $28.7 \pm 0.1$ & $8.1 \pm 0.2$ \\
\hline
\end{tabular}

*Não Significativo; **Significativo por aplicação do Tukey Test $(\mathrm{P}<0.05)$

Legenda: (T1 - náuplios de Artemia, T2 - Metanáuplios Artemia, T3 - Metanáuplios de Artemia enriquecidos com ácidos graxos, T4 - Metanáuplios de Artemia enriquecidos com ácidos graxos e Vitamina C, T5 - Dieta inerte ou artificial).

A respeito dos parâmetros de crescimento, os resultados obtidos para sobrevivência (Figura 1) indicam uma resposta positiva de larvas do jundiá apenas para os tratamentos um, dois, três e quatro, onde foram utilizados o alimento vivo na forma de náuplios e metanáuplios de Artemia.

No tratamento cinco, com uso de dieta artificial foi observada elevada mortalidade ao longo do experimento, decorrente do canibalismo, reduzindo a sobrevivência. Ademais, no decorrer do estudo, houve uma crescente redução da atividade das larvas na procura pelo alimento e em alguns momentos a ocorrência de canibalismo também foi observada, permanecendo abaixo de $10 \%$.

Para os tratamentos com $A$. salina na fase de metanáuplio, foi observado que quando fornecidos às larvas em sua forma enriquecida, com ácidos graxos e Vitamina C, promoveram maior sobrevivência durante

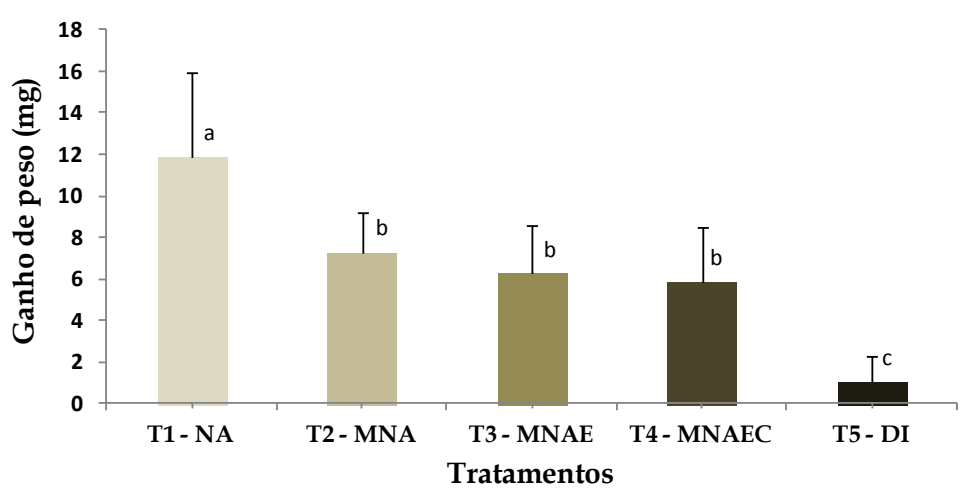

Figura 2: Ganho de peso de pós-larvas de Rhamdia quelen em relação ao alimento vivo Artêmia e dieta artificial ofertados. Legenda: (NA - náuplios de Artemia, MNA Metanáuplios Artemia, MNAE - Metanáuplios de Artemia enriquecidos com ácidos graxos, MNAEC - Metanáuplios de Artemia enriquecidos com ácidos graxos e Vitamina C, DI - Dieta inerte ou artificial). 
O fornecimento de Artemia na forma de náuplios recémeclodidos resultou na maior taxa de crescimento em ganho de peso $(P<0,05)$. Nos demais tratamentos com uso de metanáuplios, o crescimento foi menor, sendo que diferenças entre eles não foram observadas.

Assim como para qualidade de água e sobrevivência, a dieta inerte ofertada no tratamento cinco, nota-damente comprometeu o desenvolvimento das larvas. Além disso, a degradação da qualidade de água das unidades experimentais neste tratamento foi visível, pela presença de sólidos suspensos que dificultou a higienização destas.

O ganho em comprimento apresentou tendência similar ao registrado para o ganho de peso (Figura 3). O tratamento com náuplios de Artemia recém-eclodidos foi satisfatório e diferiu significativamente $(P<0,05)$. Em relação aos tratamentos com metanáuplios, estes não diferiram significativamente entre si $(P>0,05)$, mas foram inferiores ao tratamento com utilização de dieta artificial.

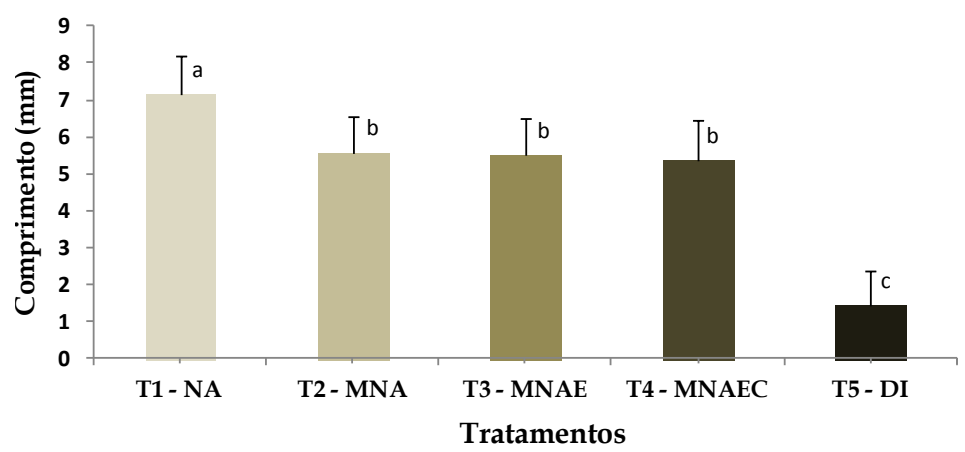

Figura 3: Variação do crescimento de pós-larvas do Rhamdia quelen entre tratamentos. Legenda: (NA - náuplios de Artemia, MNA - Metanáuplios Artemia, MNAE - Metanáuplios de Artemia enriquecidos com ácidos graxos, MNAEC Metanáuplios de Artemia enriquecidos com ácidos graxos e Vitamina C, DI - Dieta inerte ou artificial)

O efeito prejudicial da utilização de dieta artificial, comprometendo consideravelmente o crescimento das larvas de jundiá, resultando em contraste acentuado no crescimento e desenvolvimento das larvas, entre os tratamentos, pode ser observado na Figura 4.
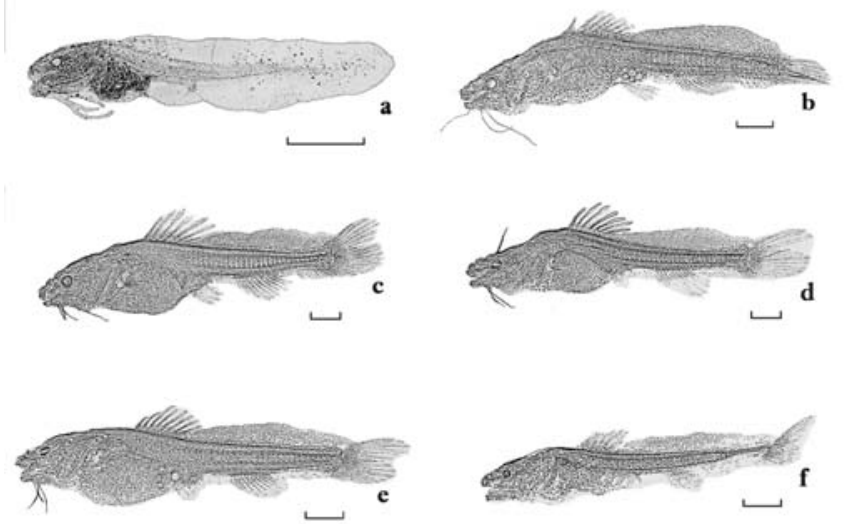

Figura 4: Contraste visual entre tratamentos para pós larvas do Rhamdia quelen. a) Pós-larva com 50 horas de vida ao inicio do experimento; b) T1 Pós-larva ao final do experimento; c) T2 - Pós-larva ao final do experimento d) T3 - Pós-larva ao final do experimento; e) T4 - Pós-larva ao final do experimento; f) T5 - Pós-larva ao final do experimento. (Escala $=1 \mathrm{~mm}$ ). Fonte: Imagens capturadas pelo autor.

\section{Discussão}

As variáveis de qualidade de água permaneceram em níveis adequados para o Jundiá (Gomes et al., 2000; Graef et al., 2007; Maffezzolli e Nuñer, 2006 e Miron et al., 2011). Apenas para a concentração de amônia total foram verificados valores acima do recomendado no tratamento cinco.

Estudos conduzidos com o jundiá, $R$. quelen, têm demonstrado a viabilidade do uso de $A$. salina na primeira alimentação como promotor de crescimento e saúde das larvas de Jundiá (Diemer et al., 2012), e do uso de ácidos graxos de diferentes fontes na melhora de sua sobrevivência (Uliana et al., 2001).

No presente estudo a utilização de $A$. salina enriquecida com ácidos graxos foi satisfatória para todos os parâmetros de desempenho avaliados. Após o processo de enriquecimento, as larvas tornaram-se mais resistentes às condições experimentais e alcançaram altos níveis de sobrevivência.

Os ácidos graxos testados por Tronco et al. (2007) em dietas artificiais foram igualmente eficientes no aumento da sobrevivência de larvas do Jundiá, R. quelen. Entretanto, não houve melhoras para o crescimento. Da mesma forma, no atual experimento, os resultados de sobrevivência foram satisfatórios e equiparáveis entre todos os tratamentos $\operatorname{com} A$. salina, mas com significativa variação em crescimento.

Embora seja satisfatório o uso de $A$. salina, quando consideramos os níveis de sobrevivência obtidos no presente estudo, houve declínio de produtividade $(\mathrm{P}<0,05)$ para o crescimento e ganho de peso no tratamento dois, três e quatro, com utilização de metanáuplios não enriquecidos e enriquecidos com ácidos graxos e vitamina $\mathrm{C}$.

Segundo Leger et al. (1986), existe a possibilidade de uma redução dos níveis proteicos em metanáuplios de $A$. salina, tendo em vista que neste estádio, metanáuplios tendem a perder valor nutricional, principalmente em proteína, devido à intensa atividade metabólica. Sendo assim, a possível deficiência em proteína apresentada por metanáuplios pode explicar as variações observadas no crescimento e ganho de peso das larvas entre os tratamentos com uso de náuplios e metanáuplios de Artemia. Em adição, observações realizada por Fracalossi et al. (2007) reforçam a suposição de que alimentos deficientes em proteína podem comprometer o desenvolvimento inicial.

Os resultados de crescimento e ganho de peso obtidos neste estudo em todos os tratamentos com o uso de alimento vivo foram similares aos observados por Carneiro et al. (2003) na larvicultura do jundiá, $R$. quelen, com emprego de zooplâncton. No entanto, a dieta inerte utilizada de forma isolada por estes autores foi igualmente danosa às larvas e resultou em menor sobrevivência em ambos os estudos.

Apesar das vantagens apresentadas pelo uso de dieta artificial (Dias, 1989), os resultados obtidos para crescimento e ganho de peso de larvas do jundiá, $R$. quelen, no presente estudo não foram satisfatórios, comparando-se com as diferentes formas de $A$. salina utilizadas. Isto sugere uma baixa capacidade de larvas do jundiá em digerir alimentos complexos contidos em dietas artificiais, assim como observado para o pacu, Piaractus mesopotamicus, considerando o baixo desenvolvimento alcançado com dieta artificial em relação ao alimento vivo (Beerli et al., 2004). 
O reduzido crescimento e sobrevivência no tratamento cinco pode ser justificado pela incapacidade morfológica e fisiológica de larvas do jundiá em digerir moléculas complexas logo após o início da alimentação exógena e durante o processo de formação do trato digestivo e comum a diversas espécies (Ronnestad et al., 1999). Ao que parece, a assimilação de dietas artificiais é restrita a fases posteriores, considerando-se que a utilização isolada de dieta artificial foi ineficiente neste experimento e igualmente insatisfatória para Behr et al. (2000) na larvicultura do jundiá. Adicionalmente, a dieta artificial foi utilizada com sucesso para R. quelen após a primeira alimentação (Carneiro et al., 2003).

A limitação de larvas do jundiá em digerir ingredientes complexos na fase inicial de vida foi comprovada por Lazzari et al. (2004). Estes autores observaram que a inclusão de glúten de milho foi ineficiente ao comprometer o desempenho das larvas. Estes resultados reforçam a dificuldade de assimilação de nutrientes complexos contidos em dietas artificiais por larvas do jundiá e estão de acordo com o fraco desempenho do tratamento cinco.

Os resultados positivos para o crescimento de larvas do jundiá obtidos neste estudo com o uso de náuplios de $A$. salina foram similares aos encontrados por Marciales-Caro et al. (2010) para o surubim cachara, $P$. fasciatum, na fase inicial de larvicultura, onde este microcrustáceo em sua forma natural foi igualmente eficiente. $A$ inclusão de vitamina $C$ no tratamento quatro não resultou em maior desempenho, comparando-se aos demais tratamentos. Da mesma forma, Reis et al. (2011) não observaram aumento no desempenho com níveis crescentes de vitamina C para Rhamdia voulezi durante a larvicultura, embora o uso combinado com outras vitaminas tenha sido reportado por Trombetta et al. (2006) como eficiente para larvas do R. quelen.

O desenvolvimento larval para o período de estudo foi compatível com a descrição morfológica de larvas do jundiá descrita por Pereira et al. (2006), embora as larvas oriundas do tratamento com dieta artificial (cinco) tenham apresentado má formação em relação aos demais tratamentos para pigmentação, formação da placa hipural, nadadeiras e sinais claros de inanição.

\section{Conclusões}

A utilização de $A$. salina em diferentes estádios de desenvolvimento e enriquecimento é eficaz em promover o crescimento e sobrevivência na fase inicial da larvicultura do jundiá, $R$. quelen. No entanto, considerando o tempo investido e dificuldades inerentes ao enriquecimento, a utilização de náuplios recém-eclodidos de $A$. salina é mais indicada.

O uso de dieta inerte comercial em fase inicial de larvicultura não é recomendado por comprometer significativamente a qualidade de água, o crescimento e sobrevivência. Além de interferir no desenvolvimento morfológico das larvas, caracterizado por quadros de inanição e má formação corporal.

\section{Agradecimentos}

Ao Conselho Nacional de Desenvolvimento Científico e Tecnológico (CNPq) pela bolsa de Mestrado concedida.

Ao programa TAXon line - Rede Paranaense de Coleções Biológicas por conceder o uso de equipamentos de captura de imagens das larvas.

Aos professores Eduardo L.C. Ballester, bolsista de produtividade do CNPq, e Leandro Portz, bolsista de produtividade da Fundação Araucária.

\section{Referências}

AGH, N.; SORGELOOS, P. Handbook of protocols and guidelines for culture and enrichment of live food for use in larviculture. UrmiaIran: Ediciones Artemia \& Aquatic Animals Research Center, Urmia University, Urmia - Iran. p. 25-41, 2005.

ARAGÃO, C.; CONCEICAO, L. E. C.; DINIS, M. T.; FYHN, H. J. Amino acid pools of rotifers and Artemia under different conditions: nutritional implications for fish larvae. Aquaculture, n. 234, p. 429445, 2004.

BEERLI, E. L.; LOGATO, P. V. R.; DE FREITAS, R. T. F. 2004. Alimentação e comportamento de larvas de pacu, Piaractus mesopotamicus (Holmberg, 1887). Cienc. Agrotecnol., Lavras,v. 28, p. 149-155, 2004.

BEHR, E. R.; RADÜNZ NETO, J.; TRONCO, A. P.; FONTANA, A. P. Influência de diferentes níveis de luminosidade sobre o desempenho de larvas de jundiá (Rhamdia quelen) (Quoy e Gaimard, 1824) (Pisces: Pimelodidae). Acta Scientiarum. Biological Sciences, v. 21, p. 325-330, 1999.

BEHR, E. R.; TRONCO, A. P.; NETO, J. R. Ação do tempo e da forma de suplementação alimentar com Artemia franciscana sobre a sobrevivência e o crescimento de larvas de jundiá. Ciência Rural, v. 30, n. 3, p. 503-507, 2000.

CARNEIRO, P. C. F.; MIKOS, J. D.; SCHORER, M.; OLIVEIRA FILHO, P. R. C.; BENDHACK, F. Live and formulated diet evaluation through initial growth and survival of jundiá larvae, Rhamdia quelen. Scientia Agricola,v. 60, p. 615-619, 2003.
CÔRTES, G de F.; TSUZUKI, M. Y. Efeito do tamanho do rotífero na sobrevivência e no crescimento de neon gobi Elacatinus figaro durante as fases iniciais de larvicultura. Bol. Inst. Pesca, São Paulo, v. 36, p. 205-212, 2010.

DIAS, T. C. R. Desenvolvimento de larvas de pacu (Piaractus mesopotamicus, Holmberg, 1887) com dietas naturais e artificiais. Jaboticabal, 1989. 53 p. Dissertação (Mestrado em Zootecnia) - Faculdade de Ciências Agrárias e Veterinárias/Jaboticabal, Universidade Estadual Paulista, 1989.

DIEMER, O.; NEU, D. H.; SARY, C.; FINKLER, J. K.; BOSCOLO, W. R.; FEIDEN, A. Artemia sp. na alimentação de larvas de jundiá (Rhamdia quelen). Ciência Animal Brasileira (Brazilian Animal Science), v. 13, p. 175-179, 2012.

FEIDEN, A.; HAYASHI, C.; BOSCOLO, W. R.; REIDEL, A. Desenvolvimento de larvas de Steindachneridion sp. em diferentes condições de refúgio e luminosidade. Pesq. agropec. bras., Brasilia, v. 41, p. 133-137, 2006.

FRACALOSSI, D. M.; BORBA, M. R.; OLIVEIRAFILHO, R. O mito da onivoria do jundiá. Panorama da Aqüicultura, v. 100, n. 1, p. 36-40, 2007.

GOMES, L. C.; GOLOMBIESKI, J. I.; GOMES, A. R. C.; BALDISSEROTTO, B. Biology of Rhamdia quelen (Teleostei, Pemelodidae). Ciência Rural, v. 30, p. 179-185, 2000.

GRAEF, A.; TOMAZON, A. F.; NAZARENO, P. E.; MARAFON, T. A. Influência da dureza e do $\mathrm{pH}$ no desenvolvimento do jundiá (Rhamdia quelen) na fase de fertilização até a produção de póslarvas. REDVET, v. 8, p. 339-347, 2007. 
HOLT, J. 2011. Larval Fish Nutrition. Wiley Online Library. Oxford. $430 \mathrm{p}$.

IHERING, R. V. A method for inducing fish to spawn. Progr. FishCult., v. 34, p. 15-16, 1937.

LAVENS, P.; SORGELOOS, P.. Manual on the production and use of live food for aquaculture. FAO. Roma. 295 p., 1996.

LAZZARI, R.; RADÜNZ NETO, J.; LIMA, R. L.; PEDRON, F. A.; LOSEKANN, M. E. Efeito da frequência de arraçoamento e da troca do tamanho de partícula alimentar no desenvolvimento de pós-larvas de jundiá (Rhamdia quelen). Revista Brasileira de Agrociência, v. 10, n. 2, p. 231-234, 2004.

LEGER, P.; BENGTSON, D.; SIMPSON, K.; SORGELOOS, P. The use and nutritional value of Artemia as a food source. Oceanography and marine biology: an annual review, 521-623, 1986.

LUZ, R. K.; PORTELLA, M. C. 2005. Freqüência alimentar na larvicultura do trairão (Hoplias lacerdae). R. Bras. Zootec, v. 34, p. $1442-1448$.

LUZ, R. K.; ZANIBONI-FILHO, E. Utilização de diferentes dietas na primeira alimentação do mandi-amarelo (Pimelodus maculatus, Lacépède). Acta Scientiarum. Biological Sciences, v. 23, p. 483489, 2008.

MAFFEZZOLLI, G.; NUÑER, A. Crescimento de alevinos de jundiá, Rhamdia quelen (Pisces, Pimelodidae), em diferentes concentrações de oxigênio dissolvido. Acta Scientiarum. Biological Sciences, v. 28, p. 41-45, 2006.

MARCIALES-CARO, L. J.; DÍAZ OLARTE, J. J.; MEDINA ROBLES, V. M.; CRUZ CASALLAS, P. E. 2010. Evaluación del crecimiento y sobrevivencia de larvas de bagre rayado Pseudoplatystoma fasciatum (Linneaus, 1766) alimentadas con alimento vivo natural y enriquecido con ácidos grasos. Revista Colombiana de Ciencias Pecuarias, v. 23, p. 308-316.

MIRON, D. S.; BECKER, A. G.; LORO, V. L.; BALDISSEROTTO, B. 2011 Waterborne ammonia and silver catfish, Rhamdia quelen: survival and growth. Ciência Rural, v. 41, p. 349-353, 2011.

PEDREIRA, M.; SANTOS DOS J. S. E.; FERREIRA, F.; SILVA, J. Efeito do tamanho da presa e do acréscimo da ração na larvicultura de pacamã. R Bras Zootec, v. 37, p. 1144-1150, 2008.
PEREIRA, C.; BARCELLOS, L.; KREUTZ, L.; QUEVEDO, R.; RITTER, F.; SILVA, L. Embryonic and larval development of jundiá (Rhamdia quelen, Quoy \& Gaimard, 1824, Pisces, Teleostei), a South American catfish. Brazilian Journal of Biology, v. 66, p. 10571063, 2006.

RADÜNZ NETO, J. Desenvolvimento de técnicas de reprodução e manejo de larvas de jundiá Rhamdia quelen. Santa Maria, 1981. 77 f. Dissertação (Mestrado em Zootecnia) - Curso de PósGraduação em Zootecnia, Universidade Federal de Santa Maria, 1981.

REIS, E. S.; FEIDEN, A.; SIGNOR, A.; ZAMINHAN, M.; FINKLER, J. K.; BOSCOLO, W. R. Suplementação de vitamina $C$ na dieta para larvas de jundiá Rhamdia voulezi. Ciência Animal Brasileira, v. 12, p. 83-89, 1981

RONNESTAD, I.; THORSEN, A.; FINN, R. N. Fish larval nutrition: a review of recent advances in the roles of amino acids. Aquaculture, v. 177, p. 201-216, 1999.

SILFVERGRIP, A. M. A systematic revision of the Neotropical catfish genus Rhamdia (Teleostei, Pimelodidae), Department of Zoology. University of Stockholm, 1996.

TROMBETTA, C. G.; NETO, R.; LAZZARI, R. Suplementação vitamínica no desenvolvimento de larvas de jundiá (Rhamdia quelen). Ciênc Agrotec, v. 30, p. 1224-1229, 2006.

TRONCO, A. P.; RADÜNZ NETO, J.; MEDEIROS, T.; LIMA, R. Alimentação de larvas de jundiá (Rhamdia quelen) com dietas semipurificadas e fontes lipídicas. Boletim do Instituto de Pesca, v. 33, p. 9-17, 2007.

ULIANA, O.; SILVA, J. H. S. D.; RADÜNZ NETO, J. Diferentes fontes de lipídios testadas na criação de larvas de jundia (Rhamdia quelen), pisces, pimelodidae; Different sources of fat tested on larvae culture of jundia (Rhamdia quelen), pisces, pimelodidae. Ciênc. rural, v. 31, p. 129-133, 2001.

ZANIBONI FILHO, E.; BARBOSA, N. C. Priming hormone administration to induce spawning of some Brazilian migratory fish. Revista Brasileira de Biologia, v. 56, p. 655-659, 1996. 